\title{
Increased Bone Morphogenetic Protein Signaling in the Cutaneous Vasculature of Patients with Calciphylaxis
}

\author{
Sagar U. Nigwekar ${ }^{a}$ Pawina Jiramongkolchai ${ }^{\mathrm{b}}$ Florian Wunderer $^{\mathrm{c}}$ Emily Bloch $^{\mathrm{c}}$ \\ Rika Ichinose $^{c}$ Rosalynn M. Nazarian ${ }^{d}$ Ravi I. Thadhani ${ }^{a}$ Rajeev Malhotra ${ }^{e}$ \\ Donald B. Bloch ${ }^{c, f}$ \\ a Division of Nephrology, Department of Medicine, Massachusetts General Hospital and Harvard Medical School, \\ Boston, MA, USA; ${ }^{b}$ Department of Otolaryngology-Head and Neck Surgery, Washington University in St. Louis, St. Louis, \\ MO, USA; ' $A$ Anesthesia Center for Critical Care Research, Department of Anesthesia, Critical Care and Pain Medicine, \\ Massachusetts General Hospital and Harvard Medical School, Boston, MA, USA; ${ }^{d}$ Pathology Service, Dermatopathology

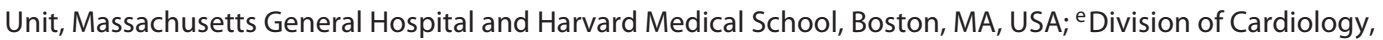 \\ Department of Medicine, Massachusetts General Hospital and Harvard Medical School, Boston, MA, USA; ${ }^{\mathrm{f} D i v i s i o n}$ \\ of Rheumatology, Allergy and Immunology, Department of Medicine, Massachusetts General Hospital and Harvard \\ Medical School, Boston, MA, USA
}

\section{Keywords}

Calcific uremic arteriolopathy · Dialysis · Inhibitor of DNA 1 . Inhibitor of DNA 3 - Phosphorylated-SMAD · Runx2 .

Vascular calcification

\begin{abstract}
Background: The objective of this study was to investigate the role of bone morphogenetic protein (BMP) signal transduction in the pathogenesis of calciphylaxis. Methods: Skin biopsy specimens were obtained from 18 patients with, and 12 patients without, calciphylaxis. Tissue sections were stained with antibodies directed against BMP effector proteins phosphorylated-SMAD ( $p$-SMAD) 1/5/9, inhibitor of DNA 1 (Id1), inhibitor of DNA 3 (Id3), and Runx2. The intensity of staining was scored semi-quantitatively as strong versus weak or absent. Results: Of the 18 patients with calciphylaxis (mean age: $59 \pm 8$ years), 9 were women and 15 had
\end{abstract}

() 2017 S. Karger AG, Basel

E-Mail karger@karger.com

www.karger.com/ajn end-stage renal disease. Of the 12 control patients (mean age: $57 \pm 10$ years), 8 were women and 8 had end-stage renal disease. Strong staining for P-SMAD 1/5/9 was detected in blood vessels from all calciphylaxis patients. In 1 patient with calciphylaxis, strong staining for $\mathrm{p}-\mathrm{SMAD}$ 1/5/9 was detected in a blood vessel that did not have evidence of calcification. Id1 and Id 3 immunoreactivity was detected in blood vessels from all 12 patients with calciphylaxis that were tested. Runx2 staining was detected in all 6 patients with calciphylaxis who were tested. p-SMAD 1/5/9 immunoreactivity was weak or absent in blood vessels of 10 of the 12 control samples. Conclusions: The BMP signal transduction pathway is activated in the cutaneous vasculature of calciphylaxis patients. The ability to detect P-SMAD 1/5/9, Id 1, and Id3 in cutaneous vasculature may assist in the diagnosis of calciphy-

S.U.N., P.J., and F.W. contributed equally to this article. 
laxis. As BMP signaling inhibitors become available, this pathway may serve as a future therapeutic target for calciphylaxis.

(c) 2017 S. Karger AG, Basel

\section{Introduction}

Calciphylaxis is a life-threatening cutaneous and subcutaneous vasculopathy characterized by calcification of small- to medium-sized arterial vessels of the dermis with intimal hyperplasia leading to vessel occlusion and skin necrosis [1,2]. Patients with calciphylaxis develop painful skin lesions (nodules, livedo reticularis, or plaques) that ulcerate and often become infected. Calciphylaxis usually affects patients with advanced kidney disease. Other risk factors that predispose to calciphylaxis include female gender, white race, obesity, diabetes mellitus, abnormalities in mineral bone metabolism, and warfarin use [3-7]. The pathogenesis of calciphylaxis is poorly understood and no treatment has been proven to be effective $[8,9]$. The prognosis for calciphylaxis is poor, with 1-year mortality estimated at $60 \%[10,11]$.

Bone morphogenetic protein (BMP) signaling is an important mediator of calcification of large and mid-sized arteries in atherosclerosis [12-15]. BMPs are members of the transforming growth factor superfamily and act by binding to heteromeric complexes of BMP type I and type II serine-threonine kinase cell surface receptors $[16,17]$. BMP ligand-binding induces BMP type II receptors to phosphorylate BMP type I receptors, which in turn phosphorylate the cytosolic BMP effector proteins, SMADs 1, 5, and 9 (SMAD 9 was previously designated SMAD 8). Phosphorylated SMAD 1, 5, or 9 translocates to the nucleus together with SMAD 4 where the heterodimer activates specific target genes, including the inhibitor of DNA (Id) binding genes 1 and 3. BMP signaling also increases the level of Runx2, an osteogenic transcription factor [18]. Increased levels of phosphorylated-SMAD (p-SMAD) 1/5/9, Id1, Id3, and Runx2 serve as markers of activation of the BMP signal transduction pathway. Extracellular antagonists of BMP ligands such as noggin, chordin, matrix Gla protein (MGP), and follistatin can sequester BMP ligands, inhibit BMP signaling, and reduce downstream activation p-SMAD 1/5/9, Id1, and Id3 [19].

We hypothesized that BMP signaling is a stimulus for vascular calcification in patients with calciphylaxis. In previous studies, Kramann et al. [20] and Tian et al. [21] detected upregulation of BMP-2, a BMP receptor ligand, in patients with calciphylaxis. Griethe et al. [22] detected increased levels of BMP-4 in cutaneous arterioles of a patient with calciphylaxis. Although, the levels of BMP agonists in calciphylaxis may be increased, the expression of MGP, one of the endogenous inhibitors of BMP signaling, is also altered in calciphylaxis patients [20,21]. Therefore, the net effects of increased BMP levels on the BMP signal transduction pathway need to be determined. We designed the present study to investigate the role of BMP signal transduction in the pathogenesis of calciphylaxis.

\section{Methods}

This study was approved by the Partners Institutional Review Board (Protocol ID: 2009P001680) and was conducted according to the principles expressed in the Declaration of Helsinki. Skin specimens were obtained during surgical procedures (including skin biopsy, debridement, and amputation) conducted for diagnostic or therapeutic indications. Clinical data were obtained by chart review.

The diagnosis of calciphylaxis was based on the presence of painful skin lesions and subcutaneous and/or dermal vascular calcification in a skin biopsy specimen. To identify potential cases of calciphylaxis, we applied an algorithm that included a billing code for "other disorders of calcium metabolism" and a skin biopsy procedure billing code. This algorithm was applied to our institute's research database. The medical records of patients identified with this algorithm were reviewed, and patients with painful skin lesions were selected. Archived skin pathology specimens from these patients were reviewed by a dermatopathologist who evaluated hematoxylin and eosin, and von Kossa stained sections on the available specimens. The controls were randomly selected from both end-stage renal disease (ESRD) and non-ESRD patients for whom archived skin specimens were available but the criteria for calciphylaxis used in this study (painful skin lesions and subcutaneous and/or dermal vascular calcification) were not met.

To detect vascular calcification, formalin-fixed, paraffin-embedded skin tissue sections were treated according to the von Kossa staining method. The von Kossa method uses a precipitation reaction in which silver ions react with phosphate contained within calcium hydroxyapatite [23]. Sections measuring $5 \mu$ were deparaffinized and placed in 5\% silver nitrate solution (Fisher Scientific, Fair Lawn, NJ, USA). The slides were exposed to light (100 $\mathrm{W}$ ) for $1 \mathrm{~h}$, rinsed, placed in 5\% sodium thiosulfate (American MasterTech, Lodi, CA, USA), and counterstained with nuclear fast red (American MasterTech, Lodi, CA, USA).

Staining of formalin-fixed, paraffin-embedded slides for $\mathrm{p}$ SMAD 1/5/9, Id 1, Id 3, and Runx2 required an antigen retrieval step, which consisted of boiling the slides in sodium citrate buffer $(\mathrm{pH}=$ $6.0,10 \mathrm{mM}$ ) for $10 \mathrm{~min}$. Tissue sections were incubated with primary antibodies and subsequently with biotinylated secondary antibody using the HistoMouse-SP kit (Invitrogen, Carlsbad, CA, USA). The presence of immune complexes was detected using the AEC (3-amino-9-ethylcarbazole) substrate-chromogen mixture, as directed by the manufacturer. Slides were counterstained with hematoxylin. Staining without a primary antibody served as a negative control. The following primary antibodies were used for immunohistochemistry: rabbit monoclonal anti-p-SMAD antibody (Maine 
Table 1. Characteristics of calciphylaxis cases and controls

\begin{tabular}{|c|c|c|c|c|c|c|c|c|c|c|}
\hline & $\begin{array}{l}\text { Age, } \\
\text { years }\end{array}$ & Gender & Race & ESRD & $\begin{array}{l}\text { ESRD duration, } \\
\text { months }\end{array}$ & $\begin{array}{l}\text { Diabetes } \\
\text { mellitus }\end{array}$ & $\begin{array}{l}\text { Serum calcium, } \\
\mathrm{mg} / \mathrm{dL}\end{array}$ & $\begin{array}{l}\text { Serum phosphate, } \\
\mathrm{mg} / \mathrm{dL}\end{array}$ & $\begin{array}{l}\text { Serum parathyroid } \\
\text { hormone, } \mathrm{pg} / \mathrm{mL}\end{array}$ & Warfarin \\
\hline \multicolumn{11}{|c|}{ Cases } \\
\hline 2 & 69 & Female & White & Yes & 29 & Yes & 9.7 & 3.5 & 129 & Yes \\
\hline 3 & 66 & Male & White & Yes & 10 & Yes & 9.6 & 3.2 & 151 & Yes \\
\hline 4 & 55 & Male & White & Yes & 151 & No & 8.6 & 4.6 & 2,140 & Yes \\
\hline 7 & 59 & Female & White & Yes & 159 & Yes & 9.0 & 3.6 & 624 & No \\
\hline 8 & 78 & Male & Black & Yes & 67 & No & 8.7 & 6.2 & 235 & Yes \\
\hline 9 & 59 & Female & Black & Yes & 48 & Yes & 8.5 & 3.5 & 720 & Yes \\
\hline 10 & 65 & Male & White & Yes & 43 & No & 10.7 & 3.4 & 341 & Yes \\
\hline 11 & 63 & Female & White & No & Not applicable & No & 9.4 & 3.9 & 31 & No \\
\hline 12 & 55 & Male & White & No & Not applicable & No & 9.8 & 3.8 & 61 & No \\
\hline 17 & 49 & Male & White & Yes & 4 & Yes & 9.6 & 3.8 & 75 & No \\
\hline 18 & 64 & Female & White & Yes & 45 & Yes & 10.3 & 4.7 & 513 & Yes \\
\hline \multicolumn{11}{|c|}{ Controls } \\
\hline 1 & 58 & Female & White & No & Not applicable & No & 9.6 & 3.3 & Not available & No \\
\hline 2 & 44 & Female & White & No & Not applicable & No & 9.5 & 2.8 & Not available & No \\
\hline 3 & 41 & Female & White & No & Not applicable & No & 9.1 & 2.5 & 145 & No \\
\hline 4 & 59 & Male & White & Yes & 60 & Yes & 8.1 & 6.1 & 694 & Yes \\
\hline 5 & 60 & Female & White & Yes & 234 & No & 8.3 & 3.1 & 196 & No \\
\hline 6 & 57 & Female & White & Yes & 56 & Yes & 9.9 & 4.4 & 463 & No \\
\hline 7 & 43 & Female & White & Yes & 3 & No & 10.1 & 3.3 & 679 & Yes \\
\hline 8 & 62 & Male & White & Yes & 58 & No & 9.7 & 6.0 & 872 & No \\
\hline
\end{tabular}

ESRD, end-stage renal disease.

Medical Center Research Institute), which reacts with p-SMADs 1, 5, and 9; rabbit monoclonal anti-Id-1 antibody (BioCheck Inc., Foster City, CA, USA), rabbit polyclonal anti-Id3 antiserum (Santa Cruz Biotechnology, Santa Cruz, CA, USA), rat monoclonal anti-Runx2 antibody (Novus Biologicals, Littleton, CO, USA), and mouse monoclonal anti- $\alpha$-smooth muscle actin antibody (Sigma-Aldrich, St. Louis, MO, USA). All primary antibodies were diluted 1:500 in phosphate buffered saline prior to use. The intensity of staining was scored semi-quantitatively as strong versus weak or absent.

\section{Results}

Clinical data regarding study patients are presented in Table 1 . Of the 18 patients with calciphylaxis (mean age: $59 \pm 8$ years), 9 were women, 10 were using warfarin, and
15 had end-stage renal disease. Of the 12 control patients (mean age: $57 \pm 10$ years), 8 were women, 3 were treated with warfarin, and 8 had end-stage renal disease.

Using von Kossa stain, calcification was detected in the medial layer of arterioles in the skin tissue specimens from all patients with calciphylaxis. Strong p-SMAD $1 / 5 / 9$ staining was detected in arterioles in all 18 calciphylaxis patients, including those with (Fig. 1a-d), and without (Fig. 2a-d), end-stage renal disease. In 1 specimen from a patient with calciphylaxis, strong p-SMAD 1/5/9 was detected in an arteriole, even though von Kossa staining of the same arteriole (in an adjacent section) failed to detect calcification (Fig. 2e-h). In patients with calciphylaxis, strong p-SMAD 1/5/9 staining was detected in blood vessels of various sizes ranging from the relatively 
Fig. 1. High levels of p-SMAD 1/5/9 were detected in the medial layer and adjacent cells of arterioles in patients with uremic calciphylaxis. A von Kossa (vK)-positive arteriole in a patient with calciphylaxis is shown at low (a) and high (b) magnification. Strong nuclear expression of p-SMAD $1 / 5 / 9$ was detected in cells in the corresponding vessel $(\mathbf{c}, \mathbf{d})$. Weak or absent $\mathrm{p}$ SMAD 1/5/9 expression was present in arterioles of control skin samples $(\mathbf{e}, \mathbf{f})$. Black boxes in $(\mathbf{a}, \mathbf{c}, \mathbf{e})$ show areas that are magnified in (b, d, f). Black bars in $(\mathbf{a}, \mathbf{c}, \mathbf{e})$ indicate $100 \mu \mathrm{m}$. Black bars in $(\mathbf{b}, \mathbf{d}, \mathbf{f})$ indicate $20 \mu \mathrm{m}$.

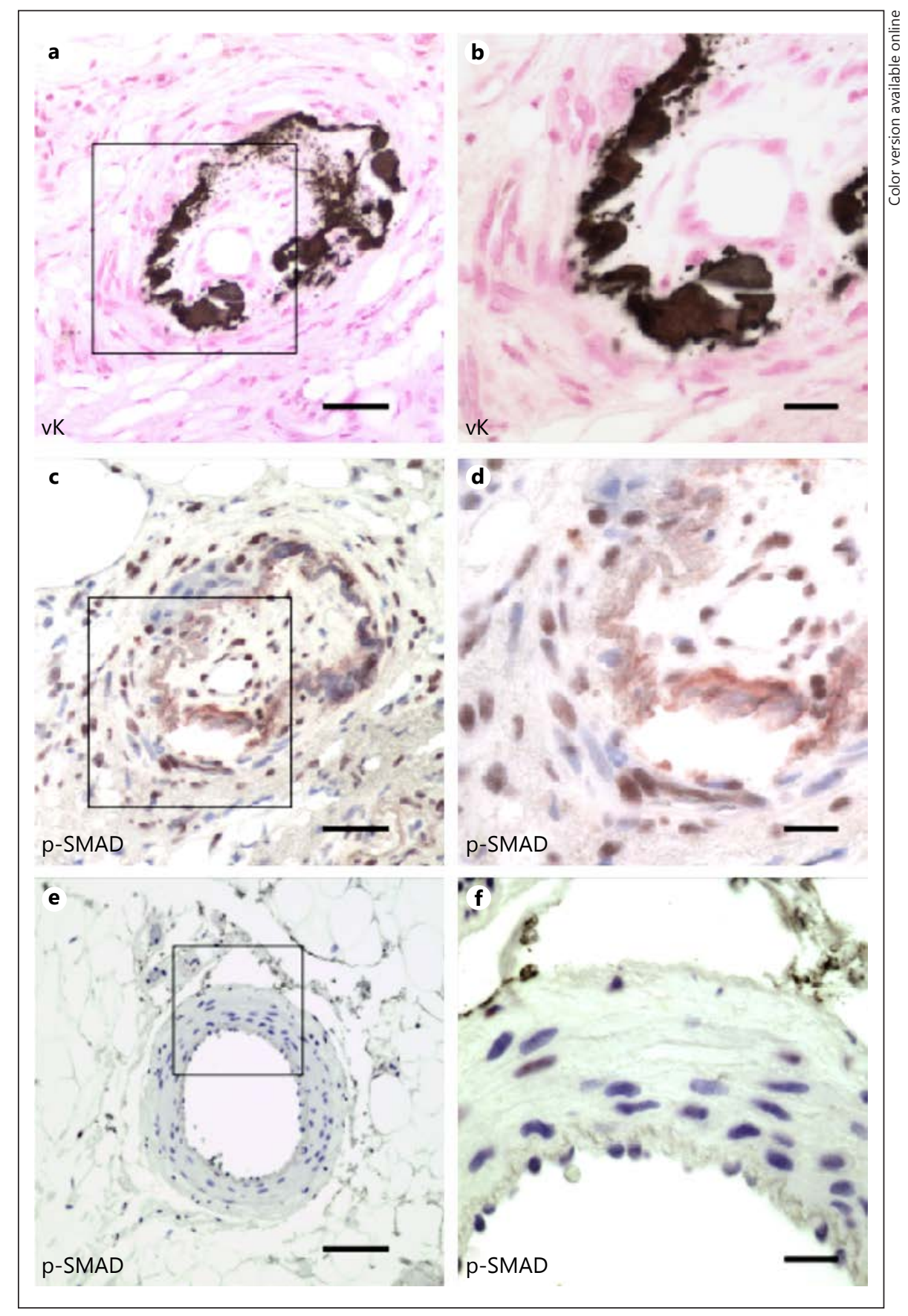

large subcutaneous arterioles to the cells lining smaller arterioles passing through adipose tissue (Fig. $3 \mathrm{a}-\mathrm{d}$ ). To determine the cell types in blood vessels that contained increased levels of p-SMAD 1/5/9, adjacent sections were stained with anti-p-SMAD 1/5/9 or anti-smooth muscle actin (SMA) antibody (Fig. 3e-h). Anti-SMA antibodypositive, smooth muscle cells in the medial layer of blood vessels stained positively for p-SMAD 1/5/9. Anti-SMA antibody-negative cells in the hyperplastic intimal layer of the blood vessels also stained positively for p-SMAD $1 / 5 / 9$. The results show that both medial layer smooth muscle cells and intimal layer SMA-negative cells have evidence of increased BMP signal transduction.

In sections from the 12 calciphylaxis patients who were tested for Id 1 and Id 3 , a distribution of staining similar to that of p-SMAD 1/5/9 (Fig. 4a, b) was noted for 
Fig. 2. High levels of p-SMAD 1/5/9 were detected in the arterioles and adjacent cells in tissue sections from 2 non-uremic calciphylaxis patients. Von Kossa (vK) staining (a, b) and p-SMAD 1/5/9 staining of the corresponding vessel $(\mathbf{c}, \mathbf{d})$ are shown. Sectioning of the tissue resulted in artifactual changes in the medial layer of the calcified vessel wall in $\mathbf{a}$; the location of the intimal layer and remaining blood vessel lumen are indicated by a blue, dashed circle. In (c), there is complete loss of the medial and intimal portion of the same vessel. The blue dashed circle indicates the expected location of the blood vessel lumen, corresponding to the adjacent tissue section shown in a. In tissue sections from one patient with calciphylaxis, von Kossa (vK) staining was negative $(\mathbf{e}, \mathbf{f})$, while an adjacent section containing the same vessel stained strongly for the presence of p-SMAD 1/5/9 $(\mathbf{g}, \mathbf{h})$. Given the size and appearance of the blood vessel, it was unlikely that the absence of calcification was an artifact of sample processing. Black boxes in $(\mathbf{a}, \mathbf{c}, \mathbf{e}, \mathbf{g})$ indicate the magnified regions shown in $(\mathbf{b}, \mathbf{d}, \mathbf{f}, \mathbf{h})$ respectively. Arrows in $(\mathbf{d}, \mathbf{h})$ indicate the location of representative p-SMAD 1/5/9-positive cells. Black bars in $(\mathbf{a}, \mathbf{c}, \mathbf{e}, \mathbf{g})$ indicate $100 \mu \mathrm{m}$. Black bars in $(\mathbf{b}, \mathbf{d}, \mathbf{f}, \mathbf{h})$ indicate $20 \mu \mathrm{m}$.

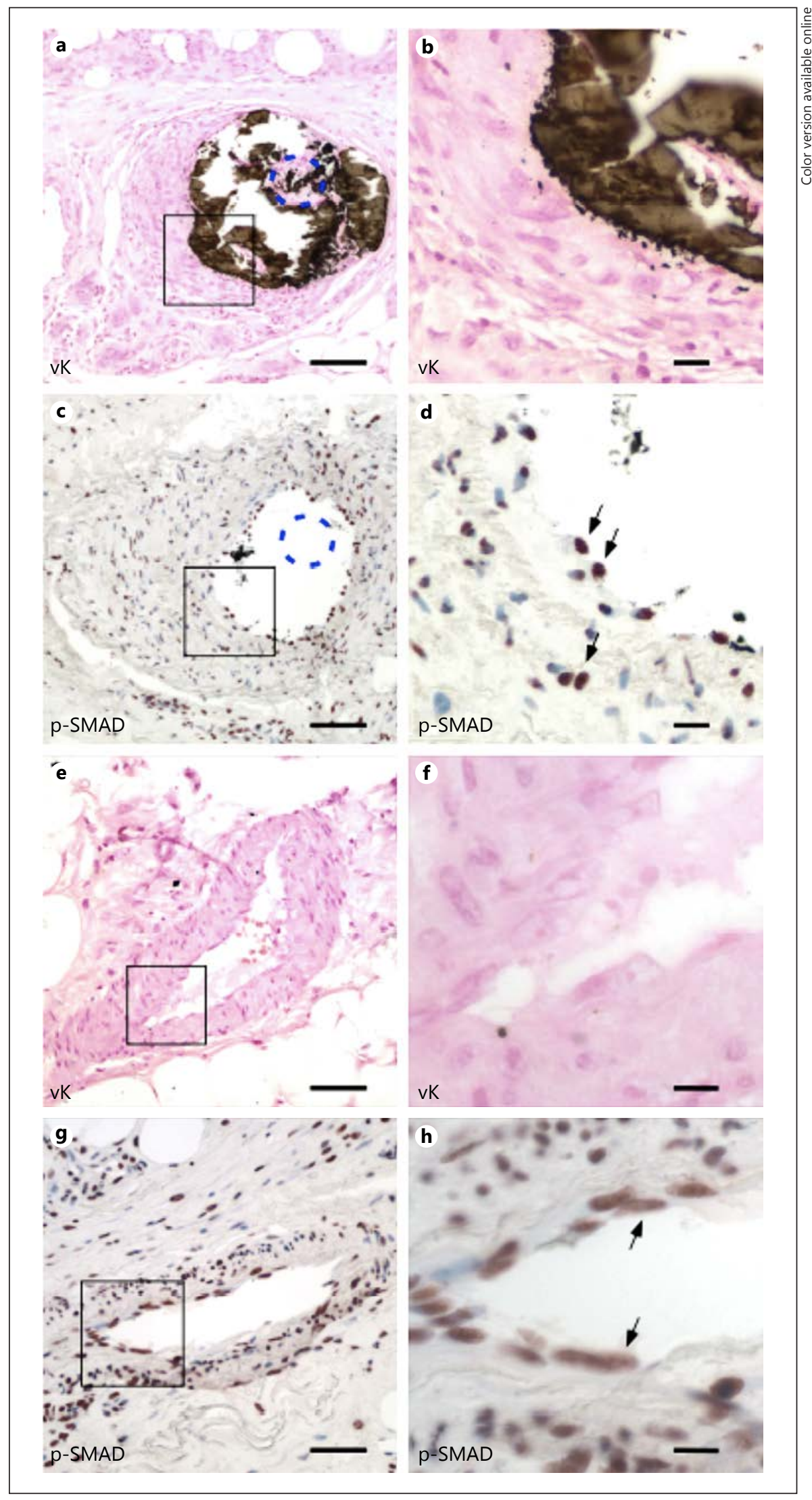

Am J Nephrol 2017;46:429-438

DOI: $10.1159 / 000484418$

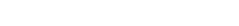


Fig. 3. p-SMAD $1 / 5 / 9$ staining was increased in arterioles of the subcutaneous fat in patients with calciphylaxis (a-d). pSMAD staining was detected in smooth muscles cells in the medial layer as well as the smooth muscle actin (SMA)-negative cells in the intima (e-h). Von Kossa staining revealed the presence of a calcified vessel in subcutaneous tissue $(\mathbf{a}, \mathbf{b})$. High levels of nuclear p-SMAD 1/5/9 were detected in cells in an adjacent section of the same vessel (c, d). Representative p-SMAD 1/5/9-positive cells are indicated by arrows in d. Anti-SMA antibody was used to indicate the location of smooth muscle cells in the arteriole in a section from a patient with calciphylaxis $(\mathbf{e}, \mathbf{f})$. Staining for p-SMAD $1 / 5 / 9$ in an adjacent section revealed nuclear p-SMAD in smooth muscle cells in the media layer (indicated by asterisks in $\mathrm{f}$ and h) as well as in SMA-negative cells in the intimal layer (indicated by arrows in $\mathbf{h}$ ). Black boxes in $(\mathbf{a}, \mathbf{c}, \mathbf{e}, \mathbf{g})$ indicate the magnified regions shown in $(\mathbf{b}, \mathbf{d}, \mathbf{f}, \mathbf{h})$ respectively. Black bars in $(\mathbf{a}, \mathbf{c}, \mathbf{e}, \mathbf{g})$ indicate 100 $\mu \mathrm{m}$. Black bars in (b, d, f, h) indicate $20 \mu \mathrm{m}$.

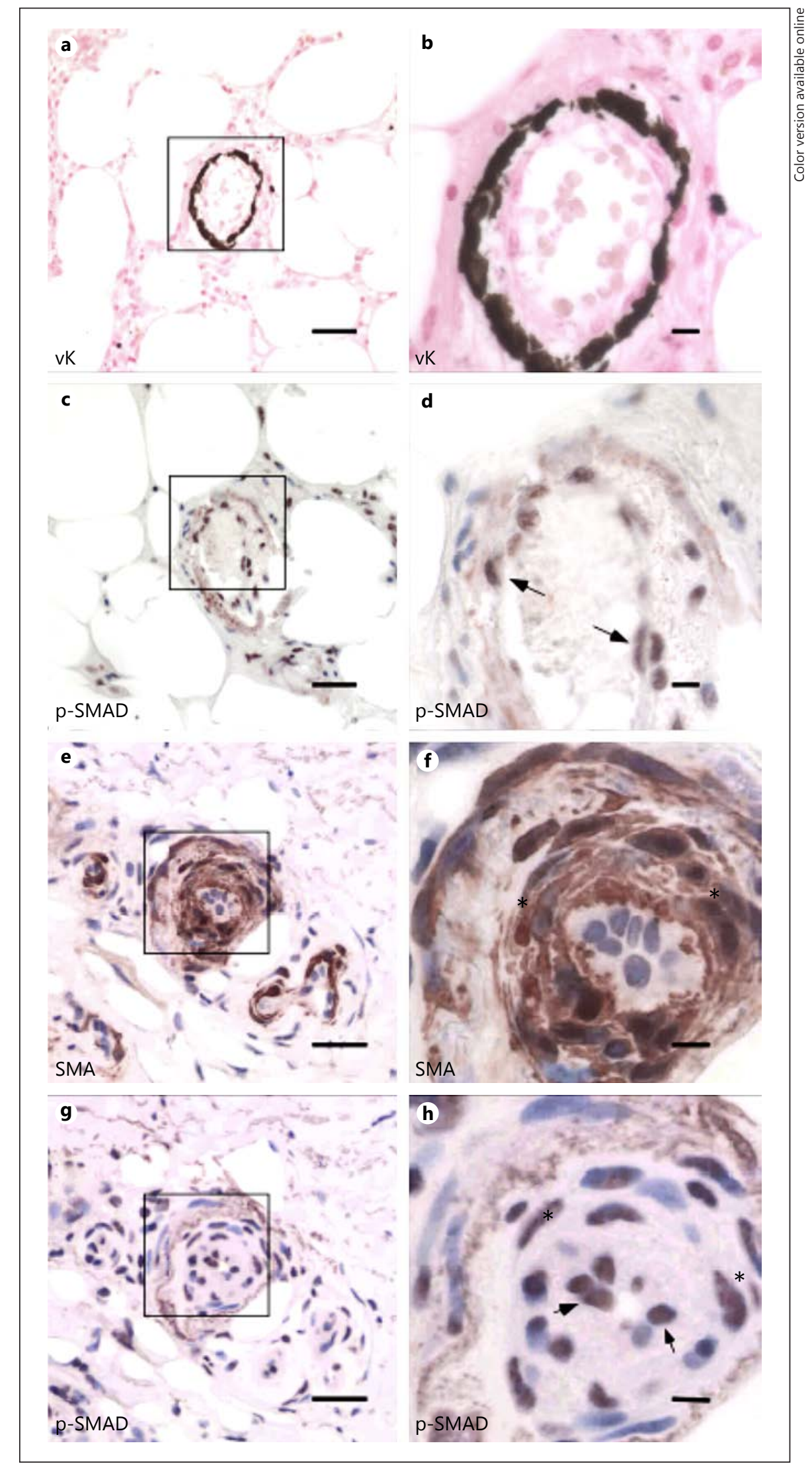


Fig. 4. Expression of Id 3 and Runx 2 was increased in arterioles in tissue sections from patients with calciphylaxis. Arterioles from a representative calciphylaxis patient stained strongly for p-SMAD 1/5/9 (a, b); an adjacent section stained strongly for the presence of $\operatorname{Id} 3$ (c, d). Strong p-SMAD $1 / 5 / 9$ staining was detected in tissue sections from a second patient with calciphylaxis (e, f); an adjacent section stained strongly for the presence of $\operatorname{Runx} 2(\mathbf{g}, \mathbf{h})$. Black arrows in (f, $\mathbf{h})$ indicate the location of representative, positively-staining cells. Black boxes in $(\mathbf{a}, \mathbf{c}, \mathbf{e}, \mathbf{g})$ indicate the magnified regions shown in $(\mathbf{b}, \mathbf{d}, \mathbf{f}, \mathbf{h})$ respectively. Black bars in $(\mathbf{a}, \mathbf{c}, \mathbf{e}, \mathbf{g})$ indicate 100 $\mu \mathrm{m}$. Black bars in (b, d, f, h) indicate $20 \mu \mathrm{m}$.

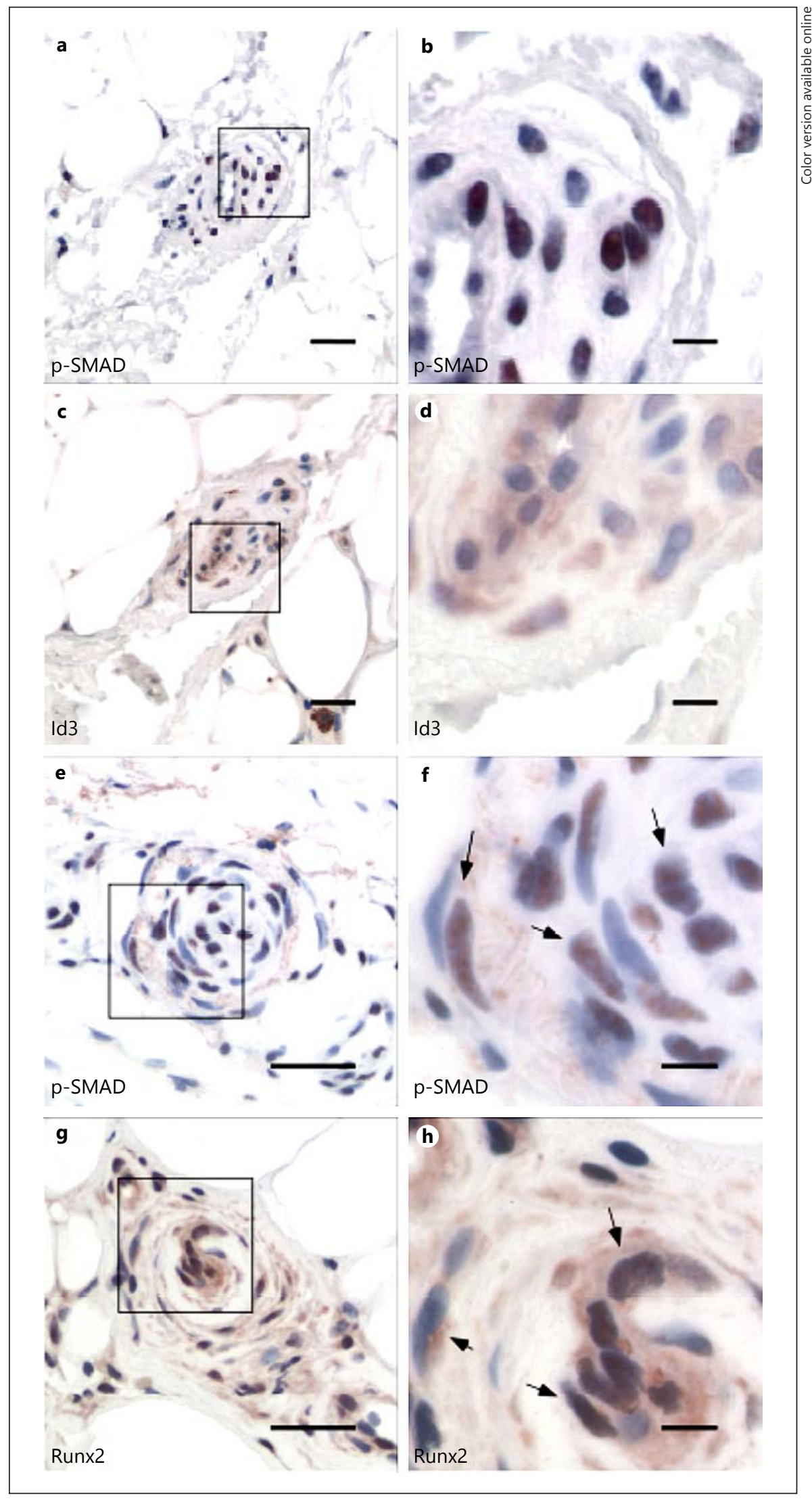

Am J Nephrol 2017;46:429-438 DOI: $10.1159 / 000484418$ 
both Id1 (not shown) and Id3 (Fig. 4c, d). As with pSMAD 1/5/9, strong Id 1 and Id 3 expression was observed in an arteriole, even though von Kossa staining of the same arteriole (in an adjacent section) failed to detect calcification (not shown). To determine whether transcription factors that are further downstream in the BMP signal transduction pathway were increased in the dermal vasculature of calciphylaxis patients, sections were stained for the presence of Runx2. Increased Runx 2 staining was detected in all 6 calciphylaxis patients who were tested for Runx2 (Fig. 4g, h).

p-SMAD 1/5/9 immunoreactivity was weak or absent in the arterioles of 10 of 12 controls (Fig. 1e, f). Staining for Id1 (not shown) and Id3 (online suppl. Figure 1a, b; for all online suppl. material, see www.karger.com/ doi/10.1159/000484418) was negative in the 6 samples that were tested. Dermal blood vessels in sections from 2 control patients had strong vascular staining for both $\mathrm{p}$ SMAD 1/5/9 and Runx2. Runx2 immunoreactivity was weak or absent in sections from the remaining 6 control patients who were tested (online suppl. Fig. 1c, d). Vascular calcification was not detected in the skin tissue specimens from 11 of 12 controls. One of the ESRD control patients had evidence of Monckeberg's medial calcinosis; however, p-SMAD 1/5/9 and Runx2 immunoreactivity was not detected in the skin tissue specimen from this control patient. Consistent with previous reports, pSMAD 1/5/9 expression was detected in some normal components of the skin, including the glandular epithelium, hair follicles, and squamous epithelium (not shown) [24].

\section{Discussion}

The results of this study show that patients with calciphylaxis have evidence of activation of the BMP signal transduction pathway in cutaneous and subcutaneous blood vessels and in the adjacent tissue. Increased levels of p-SMAD 1/5/9, Id1, Id3, and Runx2 were detected in tissue sections from patients with calciphylaxis who have end-stage renal failure and those with normal renal function, suggesting a similar disease pathogenesis.

Calciphylaxis involves calcification of the medial layer of small and medium-sized blood vessels. This distribution of calcification is similar to that seen in the MGPdeficient mouse model of vascular calcification [25]. In this model, inhibition of BMP signal transduction, using the small molecule inhibitor LDN-193189, decreased the extent of vascular calcification, suggesting that BMP signaling has an important role in disease pathogenesis [13, 15].

MGP is a potent endogenous inhibitor of vascular calcification and is thought to inhibit BMP signaling by binding and sequestering BMP ligands $[26,27]$. In a recent study, we showed that the relative plasma level of active to total (active and inactive) MGP is reduced in dialysis patients with calciphylaxis compared to dialysis patients who do not have calciphylaxis [28]. Possibly, the increase in cutaneous BMP signaling (as evidenced by increased levels of p-SMAD 1/5/9, Id1, Id3, and Runx2) observed in calciphylaxis patients in the present study can be attributed to the decreased relative level of active MGP.

In 1 patient with calciphylaxis, we detected strong $\mathrm{p}$ SMAD 1/5/9 staining in a vessel that did not have detectable calcification. This finding has potential clinical implications: the ability to detect evidence of increased BMP signaling in blood vessels with negative von Kossa staining may aid in the early diagnosis of calciphylaxis and may allow some patients to avoid repeat and deeper skin biopsies. This is particularly relevant because skin biopsies have been shown to induce or exacerbate skin ulcerations in calciphylaxis patients $[10,29]$.

Increased p-SMAD 1/5/9 and Runx2 levels were detected in sections from 2 of the control patients in this study. On further review of the medical records of these patients, both had end-stage renal disease, and calciphylaxis was considered in the differential diagnosis but was ultimately excluded due to the absence of subcutaneous or dermal vascular calcification. One patient left the hospital against medical advice and was lost to follow-up and the second died of multiple organ failure. We were therefore unable to confirm a diagnosis of calciphylaxis in these patients. There are at least 3 possible explanations for the presence of increased BMP signal transduction in 2 of the 12 control patients in this study. First, increased levels of p-SMAD 1/5/9 may be a more sensitive marker of calciphylaxis than vascular calcification. Consistent with this possibility, we observed strong P-SMAD 1/5/9 immunoreactivity in a tissue section from a calciphylaxis patient in which calcification on an adjacent section was not detected. Second, activation of BMP signaling may be a precursor to the development of calcification seen in patients with calciphylaxis. A third possibility is that other factors, including inflammation, may contribute to activation of the BMP signaling pathway. A larger study, with additional groups of control patients, will be needed to further investigate whether increased BMP signaling is a specific marker for the diagnosis of calciphylaxis. 
In conclusion, we provide evidence of increased cutaneous vascular BMP signal transduction in tissue sections from patients with calciphylaxis. The ability to detect the downstream products of BMP signaling may improve the sensitivity of skin biopsy for the diagnosis of calciphylaxis. In the future, as small molecule inhibitors of BMP signaling become available, inhibition of this pathway may serve as an effective approach to the treatment of patients with calciphylaxis.

\section{Acknowledgments}

S.U.N. is supported by American Heart Association's NCRP Winter2015Fellow-to-FacultyTransition Award(15FTF25980003) and by KL2/Catalyst Medical Research Investigator Training award (an appointed KL2 award) from Harvard Catalyst, The Harvard Clinical and Translational Science Center (National Center for Research Resources and the National Center for Advancing Translational Sciences, National Institutes of Health Award KL2 TR001100). S.U.N. also received support from National Kidney Foundation's Young Investigator Award, Fund for Medical Discovery Award from Massachusetts General Hospital's Executive Committee on Research (R00000000007190), American Heart Association's NCRP Summer 2014 Mentored Clinical and Popula- tion Research Award (15CRP22900008) to S.U.N., P.J. was supported by the Howard Hughes Medical Institute. Florian Wunderer was supported by the Deutsche Forschungsgemeinschaft (DFG Wu 841/1-1). R.I.T. is supported by National Institute of Health grants DK094872 and DK094486. R.M. was supported by the Fellow-to-Faculty Transition Award 11FTF7290032 from the American Heart Association, the Wild Family Foundation, the Hassenfeld Scholar award, and the K08HL111210 grant from the National Heart, Lung, and Blood Institute. D.B.B. is supported by the Leducq Foundation and by National Institute of Diabetes and Digestive and Kidney Diseases grant DK082971. The content is solely the responsibility of the authors and does not represent the official views of Harvard Catalyst, Harvard University and its affiliated academic healthcare centers, or the National Institutes of Health.

Preliminary findings from this manuscript were presented as a poster abstract at the 2014 American Heart Association's Scientific Sessions held in Chicago, Illinois.

\section{Disclosure Statement}

S.U.N. reports receiving speaker honorarium from SanofiAventis and has served as a consultant to Ardelyx. R.I.T. is a consultant to Fresenius Medical Care North America and Celgene, and has received a research grant from Abbott Laboratories. R.M. receives consultant fees from Akros Pharma, Inc. and Third Pole, Inc.

\section{References}

1 Brandenburg VM, Kramann R, Specht P, Ketteler M: Calciphylaxis in CKD and beyond. Nephrol Dial Transplant 2012;27: 1314-1318.

-2 Nigwekar SU, Kroshinsky D, Nazarian RM, Goverman J, Malhotra R, Jackson VA, et al: Calciphylaxis: risk factors, diagnosis, and treatment. Am J Kidney Dis 2015;66:133-146.

-3 Nigwekar SU, Zhao S, Wenger J, Hymes JL, Maddux FW, Thadhani RI, et al: A nationally representative study of calcific uremic arteriolopathy risk factors. J Am Soc Nephrol 2016; 27:3421-3429.

-4 Brandenburg VM, Kramann R, Rothe H, Kaesler N, Korbiel J, Specht P, et al: Calcific uraemic arteriolopathy (calciphylaxis): data from a large nationwide registry. Nephrol Dial Transplant 2017;1;32:126-132.

5 Hayashi M, Takamatsu I, Kanno Y, Yoshida T, Abe T, Sato Y: A case-control study of calciphylaxis in Japanese end-stage renal disease patients. Nephrol Dial Transplant 2012;27: 1580-1584.

-6 Nigwekar SU, Wolf M, Sterns RH, Hix JK: Calciphylaxis from nonuremic causes: a systematic review. Clin J Am Soc Nephrol 2008; 3:1139-1143.

7 Nigwekar SU, Bhan I, Turchin A, Skentzos SC, Hajhosseiny R, Steele D, et al: Statin use and calcific uremic arteriolopathy: a matched case-control study. Am J Nephrol 2013;37: 325-332.

-8 Brandenburg VM, Evenepoel P, Floege J, Goldsmith D, Kramann R, Massy Z, et al: Lack of evidence does not justify neglect: how can we address unmet medical needs in calciphylaxis? Nephrol Dial Transplant 2016;31: 1211-1219.

-9 Ross EA: Evolution of treatment strategies for calciphylaxis. Am J Nephrol 2011;34:460467.

10 Fine A, Zacharias J: Calciphylaxis is usually non-ulcerating: risk factors, outcome and therapy. Kidney Int 2002;61:2210-2217.

11 Sprague SM: Painful skin ulcers in a hemodialysis patient. Clin J Am Soc Nephrol 2014;9: 166-173.

-12 Derwall M, Malhotra R, Lai CS, Beppu Y, Aikawa E, Seehra JS, et al: Inhibition of bone morphogenetic protein signaling reduces vascular calcification and atherosclerosis. Arterioscler Thromb Vasc Biol 2012;32:613-622.

13 Malhotra R, Burke MF, Martyn T, Shakartzi HR, Thayer TE, O’Rourke C, et al: Inhibition of bone morphogenetic protein signal transduction prevents the medial vascular calcification associated with matrix Gla protein deficiency. PLoS One 2015;10:e117098.

14 Morrell NW, Bloch DB, ten Dijke P, Goumans MJ, Hata A, Smith J, et al: Targeting
BMP signalling in cardiovascular disease and anaemia. Nat Rev Cardiol 2016;13:106-120.

15 Yao Y, Bennett BJ, Wang X, Rosenfeld ME, Giachelli C, Lusis AJ, et al: Inhibition of bone morphogenetic proteins protects against atherosclerosis and vascular calcification. Circ Res 2010;107:485-494.

16 Cai J, Pardali E, Sánchez-Duffhues G, ten Dijke P: BMP signaling in vascular diseases. FEBS Lett 2012;586:1993-2002.

-17 Bragdon B, Moseychuk O, Saldanha S, King D, Julian J, Nohe A: Bone morphogenetic proteins: a critical review. Cell Signal 2011;23: 609-620.

18 Lin ME, Chen TM, Wallingford MC, Nguyen NB, Yamada S, Sawangmake C, et al: Runx2 deletion in smooth muscle cells inhibits vascular osteochondrogenesis and calcification but not atherosclerotic lesion formation. Cardiovasc Res 2016;pii:cvw205.

19 Balemans W, Van Hul W: Extracellular regulation of BMP signaling in vertebrates: a cocktail of modulators. Dev Biol 2002;250:231250 .

20 Kramann R, Brandenburg VM, Schurgers LJ, Ketteler M, Westphal S, Leisten I, et al: Novel insights into osteogenesis and matrix remodelling associated with calcific uraemic arteriolopathy. Nephrol Dial Transplant 2013;28: 856-868. 
-21 Tian F, Patterson AT, Davick JJ, Ing SW, Kaffenberger BH, Gru AA: The cutaneous expression of vitamin K-dependent and other osteogenic proteins in calciphylaxis stratified by clinical features and warfarin use: a case control study.JAmAcadDermatol2016;75:840-482.e1.

-22 Griethe W, Schmitt R, Jurgensen JS, Bachmann S, Eckardt KU, Schindler R: Bone morphogenic protein-4 expression in vascular lesions of calciphylaxis. J Nephrol 2003;16:728-732.

23 Puchtler H, Meloan SN: Demonstration of phosphates in calcium deposits: a modification of von Kossa's reaction. Histochemistry 1978;56:177-185.
$24 \mathrm{He} \mathrm{W}$, Cao T, Smith DA, Myers TE, Wang XJ: Smads mediate signaling of the TGFbeta superfamily in normal keratinocytes but are lost during skin chemical carcinogenesis. Oncogene 2001;20:471-483.

25 Luo G, Ducy P, McKee MD, Pinero GJ, Loyer E, Behringer RR, et al: Spontaneous calcification of arteries and cartilage in mice lacking matrix GLA protein. Nature 1997;386:78-81.

-26 Yao Y, Shahbazian A, Bostrom KI: Proline and gamma-carboxylated glutamate residues in matrix Gla protein are critical for binding of bone morphogenetic protein-4. Circulation Res 2008;102:1065-1074.
27 Schurgers LJ, Uitto J, Reutelingsperger CP: Vitamin K-dependent carboxylation of matrix Gla-protein: a crucial switch to control ectopic mineralization. Trends Mol Med 2013;19:217-226.

28 Nigwekar SU, Bloch DB, Nazarian RM, Vermeer C, Booth SL, Xu D, et al: Vitamin Kdependent carboxylation of matrix gla protein influences the risk of calciphylaxis. J Am Soc Nephrol 2017;28:1717-1722.

29 Sreedhar A, Sheikh HA, Scagliotti CJ, Nair R; Advanced-stage calciphylaxis: think before you punch. Cleve Clin J Med 2016;83:562564 